\title{
Growth of Legionella pneumophila in a human macrophage-like (U937) cell line
}

\author{
Eric Pearlman, ${ }^{1}$ Asmina H. Jiwa, ${ }^{1}$ N. Cary Engleberg ${ }^{1,2,3}$ and Barry I. \\ Eisenstein ${ }^{1,2 *}$ \\ ${ }^{1}$ Departments of Microbiology and Immunology and ${ }^{2}$ Internal Medicine, University of \\ Michigan, Ann Arbor, MI 48109-0620, U.S.A. and ${ }^{3}$ Ann Arbor Veterans Administration Hospital
}

(Received March 16, 1988; accepted April 18, 1988)

Pearlman E. (Dept. of Microbiology and Immunology, University of Michigan, Ann Arbor, MI 48109-0620, U.S.A.), A. H. Jiwa, N. C. Engleberg and B. I. Eisenstein. Growth of Legionella pneumophila in a human macrophage-like (U937) cell line. Microbial Pathogenesis 1988; 5 : 87-95.

We established a model of the bacteria-macrophage interaction to study the cellular basis of Legionella pneumophila pathogenesis and to characterize avirulent $L$. pneumophila. We found that U937 cells, which are derived from a human histiocytic lymphoma cell line, support intracellular growth of $L$. pneumophila with a doubling time of $6 \mathrm{~h}$, and that sustained intracellular growth is associated with a cytopathic effect (CPE) that can be detected by microscopic examination and quantified with the vital stain 3-(4,5-dimethyl thiazol-2-yl)-2,5,diphenyl tetrazolium bromide (MTT). An L. pneumophila isolate obtained directly from infected guinea-pig spleens can grow and produce CPE in these cells, destroying most of the cell layer after $72 \mathrm{~h}$ of growth. Only $10^{6}$ organisms of this strain are required to kill $50 \%$ of guinea-pigs inoculated by the intraperitoneal route. In contrast, an avirulent isolate derived by 203 successive plate passages of the same strain can neither kill guinea-pigs at an intraperitoneal inoculum of $10^{7}$ nor grow or produce CPE in U937 cells. Since the cells were able to differentiate between a virulent and an avirulent strain of L. pneumophila, we conclude that U937 cells are an appropriate model system for study of the bacteria-macrophage interaction

Key words: L. pneumophila; U937 cells; intracellular growth.

\section{Introduction}

During the course of Legionnaires' disease, Legionella pneumophila replicates primarily intracellularly within alveolar macrophages. ${ }^{1,2}$ To characterize the intracellular life cycle of this organism, investigators have employed a variety of host cells capable of supporting growth, including human monocytes, ${ }^{3}$ free-living protozoa, ${ }^{4-6}$ human and animal epithelial cell lines, ${ }^{7,8}$ and primary explanted macrophages from various origins..$^{9-14}$ To our knowledge, there have been no prior attempts to demonstrate intracellular growth of $L$. pneumophila in a host cell having both the properties of a human alveolar macrophage and the properties of a continuous cell line.

The purpose of this study was to determine if a cell line with these combined properties would permit the intracellular growth of $L$. pneumophila and whether this cell line could discriminate between guinea-pig virulent and avirulent derivatives of $L$.

\footnotetext{
* Author to whom correspondence should be addressed.
} 
pneumophila. Such a cell line would have the obvious advantages of ease of use, reproducibility, and relevance to native human infection. As a candidate cell model system, we chose the U937 cell line, which was derived from a human histiocytic lymphoma. ${ }^{15}$ This cell line is maintained as replicative non-adherent cells having many of the biochemical and morphological characteristics of blood monocytes. ${ }^{16}$ When treated with phorbol myristate acetate (PMA), U937 cells differentiate to become adherent, non-replicative cells with characteristics of tissue macrophages, including isoenzyme patterns, ${ }^{17}$ CR3 expression, ${ }^{18}$ and other phenotypic markers. ${ }^{16}$ Because of these properties, this cell line has been used by investigators to support the growth of other intracellular pathogens. ${ }^{19-21}$

We validated the U937 cell line as a model for L. pneumophila/macrophage interaction by demonstrating that PMA-transformed U937 cells support the intracellular growth of $L$. pneumophila bacteria. In addition, we showed that this macrophage model can distinguish guinea-pig-virulent from avirulent strains of $L$. pneumophila.

\section{Results}

\section{Kinetics of intrace/lular growth}

In preliminary experiments we confirmed that PMA-transformed U937 cells would adhere to the base of wells on polystyrene tissue culture plates and that the cells resisted displacement by vigorous washing. Using this monolayer system we next determined whether $L$. pneumophila would grow intracellularly.

We incubated L. pneumophila with U937 cell monolayers for $2 \mathrm{~h}$, at which time we removed the extracellular bacteria by washing the monolayer three times. During the logarithmic phase of growth, the bacteria doubled in number every 6 h (Fig. 1), and the infection continued until the macrophage layer was destroyed. To show that growth is dependent on the presence of intact cells, we incubated the bacteria with $5 \times 10^{5}$ U937 cells that had been disrupted by three cycles of freeze/thaw. No growth was noted under these conditions (Fig. 1), or when the bacteria were incubated

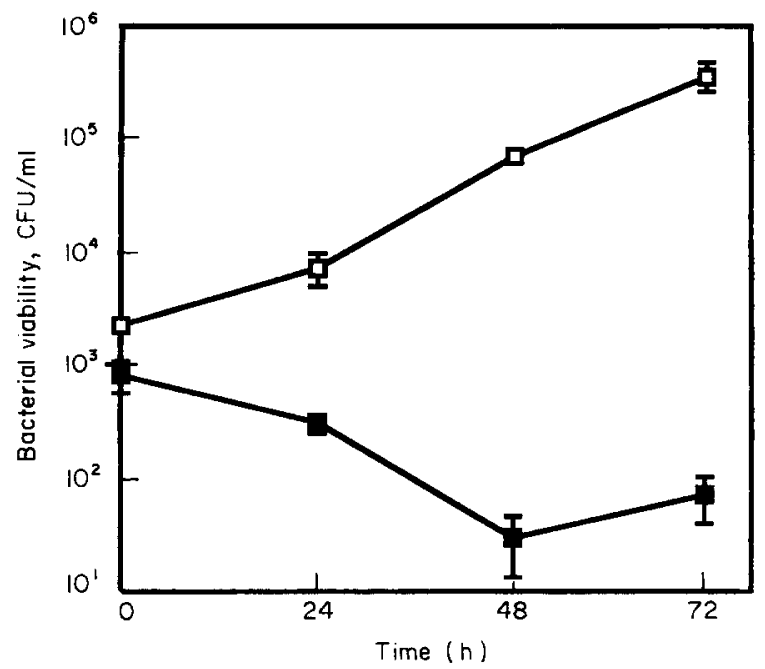

Fig. 1. Kinetics of growth of L. pneumophila in U937 cells. L. pneumophila were incubated either with live U937 cells $(\square)$ or with U937 cells that had been disrupted by freeze/thawing ( $\square$ ). At each time point, the number of viable bacteria was determined by lysing intact cells and culturing the samples on BCYE agar. 

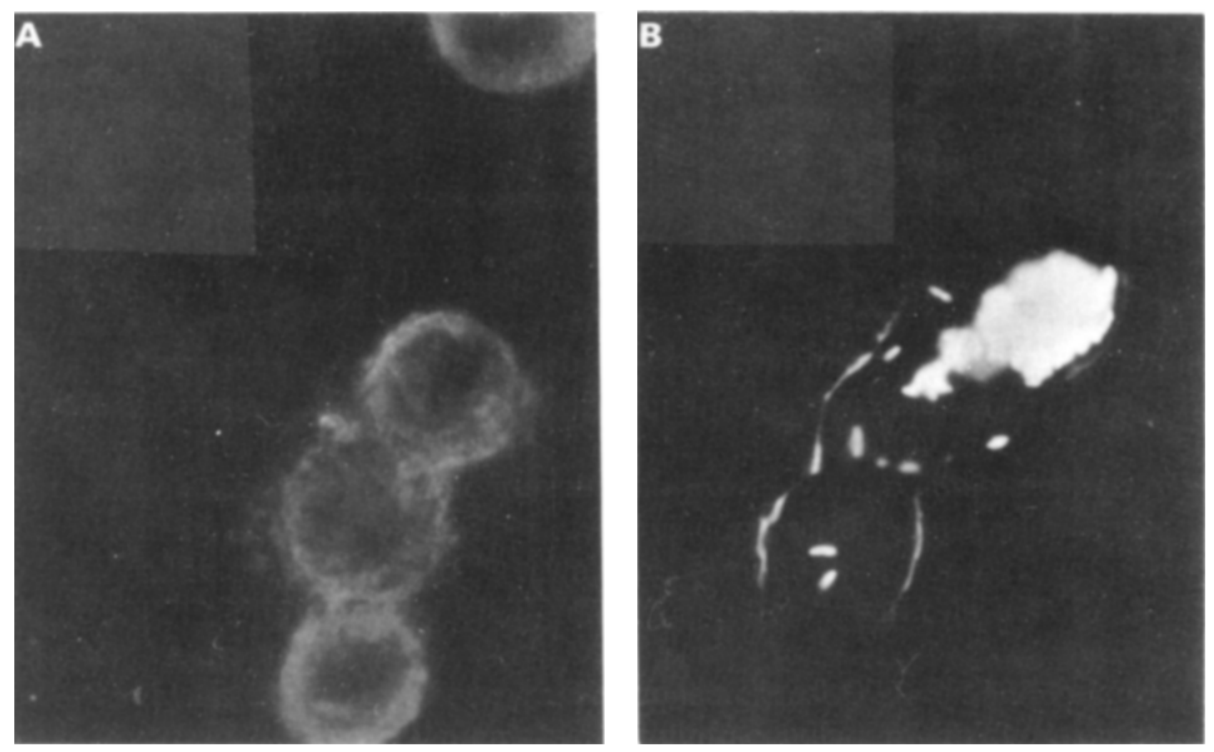

Fig. 2. Fluorescence microscopy of U937 cells infected with L. pneumophila. Infected cultures were stained with an FITC labeled anti-L. pneumophila monoclonal antibody $48 \mathrm{~h}$ after infection. The cultures were stained when live (to visualize only extracellular bacteria) (A), or after methanol fixation (to visualize both intracellular and extracellular bacteria) (B). Note that although no bacteria are visualized in the live, intact cells $(A)$, there are numerous individual bacteria associated with the four adjacent cells shown in panel B, and a large mass of fluorescent bacteria in the uppermost cell. The fluorescence of the cells themselves in panel $A$ was red in color and therefore not due to fluorescein staining. This non-specific background was diminished after methanol fixation, as can be seen in panel $B$.

with Dulbecco's Modified Eagle Medium supplemented with $10 \%$ fetal calf serum (DMEM/FCS) alone (data not shown).

To corroborate the ability of U937 cells to support the intracellular growth of $L$. pneumophila, we examined the $72 \mathrm{~h}$-infected monolayer by fluorescence microscopy after staining with an FITC-labeled anti-L. pneumophila monoclonal antibody. When we examined the living cells, which do not permit antibody penetration, we saw no bacteria associated with the cells [Fig. 2(A)]. In contrast, when the cells were first fixed with methanol to permit intracellular penetration of the antibody, we found that bacteria were clearly visible in association with the cells [Fig. 2(B)]. Thus, the organisms were present and growing within the cells.

\section{Loss of U937 cell viability during bacterial growth}

During the course of the intracellular growth experiments, we noted that the U937 cells were destroyed as the infection proceeded. Using the vital stain 3-(4,5-dimethyl thiazol-2-yl)-2,5,-diphenyl tetrazolium bromide (MTT) to quantitate the number of living cells present during the infection, we were able to measure the amount of monolayer destruction. MTT, a colorogenic substrate for mitochondrial dehydrogenase, is reduced in living cells to a purple formazan which can be quantitated spectrophotometrically. ${ }^{22}$ As described below (Materials and Methods), there is a linear correlation between MTT staining, measured at $\mathrm{OD}_{550}$, and $\mathrm{U} 937$ cell viability.

To quantitate the monolayer destruction by the growing bacteria, we incubated the organisms with the U937 cells at a ratio of 10:1 (bacteria:cells) for two hours and then killed the extracellular organisms with gentamicin sulfate, an antibiotic that does not penetrate phagocytic cells. ${ }^{23}$ The only surviving bacteria would be those sequestered within the U937 cells. After removing the antibiotic, we determined the 


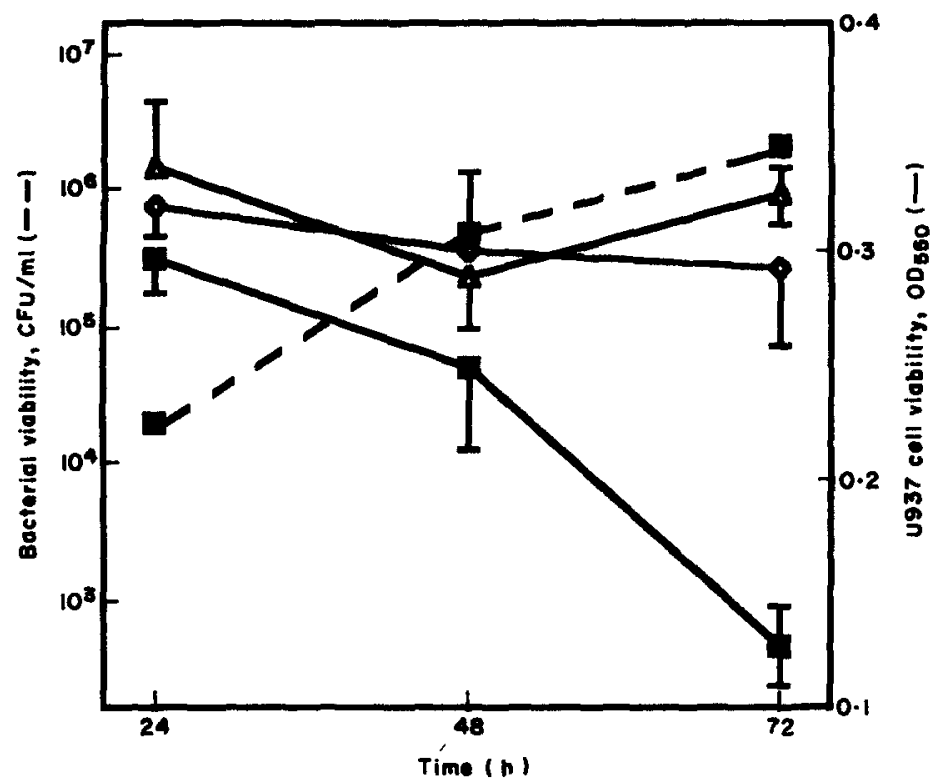

Fig. 3. Effect of intracellular growth of L. pneumophila on U937 cell viability. U937 cells were infected either with $L$. pneumophila (followed by killing of the extracellular organisms with gentamicin sulfate), with organisms that had been previously killed by $2 \mathrm{~h}$ preincubation with the antibiotic, or with no bacteria. At each time point we determined the number of viable bacteria ( $\square$ ) by plate counts, and the number of viable U937 cells remaining by measuring the $O D_{550}$ following infection with either live bacteria ( $\square$ ), killed bacteria $(\triangle)$, or no bacteria $(\diamond)$.

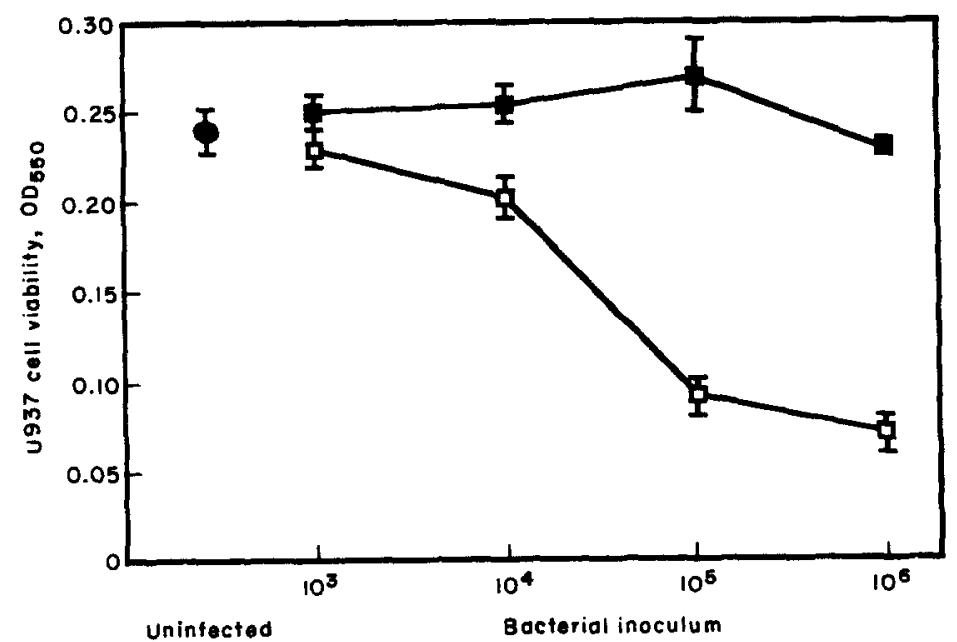

Fig. 4. Effect of L. pneumophila infection on U937 cell viability. The number of viable U937 cells remaining was determined by staining with $M T T$ and measuring $O D_{550} 48 \mathrm{~h}$ after infection with $L$. pneumophila that either were guinea-pig-passaged $(\square)$ or were passaged serially on agar 203 times ( $\square$ ). Uninfected cells $(\mathbf{O})$.

number of viable (i.e., intracellular) organisms by mild detergent lysis of the cells, and the number of viable U937 cells by vital staining. We found that the number of viable U937 cells associated with the cultures decreased over time as the bacteria increased in number (Fig. 3). In contrast, U937 cells incubated with dead L. pneumophila showed no loss in viability over this time demonstrating that host cell death is 
Table 1 Comparison of the parent L. pneumophila strain with the multiply passaged derivative.

\begin{tabular}{lcc}
\hline & \multicolumn{2}{c}{ L. pneumophila strain } \\
\cline { 2 - 3 } & $130 \mathrm{~b}$ & $130 \mathrm{~b}: 203$ \\
\hline Guinea pig virulence & $2 / 3^{a}$ & $0 / 3^{a}$ \\
Intracellular growth in U937 cells $^{b}$ & $5 \times 10^{5}$ & $\begin{array}{c}0^{2} \\
\text { Extracellular growth in yeast extract broth }\end{array}$ \\
\hline
\end{tabular}

\footnotetext{
- Number of guinea-pigs killed $3 \mathrm{~d}$ after inoculation by the intraperitoneal route with $10^{6}$ bacteria.

${ }^{b}$ CFU's after $72 \mathrm{~h}$ of infection were determined by detergent lysis of the cells followed by plate counts.

${ }^{c}$ Obtained after $16 \mathrm{~h}$ growth in yeast extract broth. During this time the cultures increased in optical density from $O D_{660}=0.15$ to $O D_{660}=0.85$ and 0.88 in the parent and $130 \mathrm{~b}: 203$, respectively.
}

dependent upon intracellular growth of L. pneumophila, and not due to the effects of a preformed bacterial toxin.

Characterization of an avirulent $\mathrm{L}$. pneumophila derivative

To demonstrate that $U 937$ cells are a relevant model of L. pneumophila pathogenicity, we investigated the capacity of the U937 cell model system to distinguish virulent from avirulent derivatives of an L. pneumophila strain. Several studies have shown that passaging $L$. pneumophila on artificial media reduces both the virulence of the organisms for guinea-pigs ${ }^{24,25}$ and their ability to grow in human monocytes. ${ }^{26}$ To attenuate a virulent strain, we passaged $L$. pneumophila strain $130 \mathrm{~b}$ on BCYE agar twice weekly for over two years (203 passages) to give rise to strain 130b:203. This agar-passaged strain was compared with the strain utilized throughout this study (strain $130 \mathrm{~b}$ recently recovered from guinea pigs) by the following parameters: (a) guinea pig virulence; (b) growth in U937 cells, and (c) growth in yeast extract broth.

We found that, in contrast to the parent strain, L. pneumophila strain 130b:203 was incapable of killing guinea-pigs after intraperitoneal inoculation (Table 1). Whereas there was no difference in the ability of the strains to grow in artificial media, the parent strain was much more capable of growing in U937 cells (Table 1) and producing CPE during intracellular growth (Fig. 4) than the long-term passaged derivative. Thus, loss of guinea-pig virulence was paralleled by loss of U937 cell virulence but not by change in growth ability in artificial media.

\section{Discussion and conclusions}

The purpose of the present study was to establish a reproducible and relevant in vitro system of $L$. pneumophila/macrophage interaction so that we can proceed to investigate the interaction between the parasite and the host at the cellular level. We have previously identified and cloned several protein antigens of $L$. pneumophila, ${ }^{27-29}$ and we plan to determine the role of these proteins in the interaction of these bacteria both with $U 937$ cells and with human alveolar macrophages. To perform these studies, we require a model of cellular infection that reflects the in vivo interaction and can be used to screen mutants that are defective in intracellular growth. For this purpose, the system of choice must: (a) support intracellular growth of virulent $L$. pneumophila; (b) fail to support the growth of avirulent L. pneumophila; and (c) reliably reproduce significant findings in subsequent experiments.

$L$. pneumophila has been grown intracellularly in a variety of other systems. ${ }^{3-14}$ 
Although explanted human alveolar macrophages would seem to be the most physiologic model available, it presents two serious problems as a screening assay: (1) the difficulty in obtaining a continuous supply of fresh cells, and (2) the variability of the cells in successive experiments (since both the donor and the state of macrophage activation would not be controllable).

The macrophage-like cell line employed in our experiments overcome these disadvantages. U937 cells are monoblastic in continuous culture, but their neoplastic nature can be reversed by various inducers such that they simultaneously lose their proliferative capacity and develop features of tissue macrophages. ${ }^{15-18}$ The advantages of such a cell line are several: (i) they are of human origin; (ii) they are available in a continuous and renewable supply; (iii) their state of activation is experimentally controlled and can therefore be standardized; and (iv) they are phenotypically similar, if not identical to macrophages, being actively phagocytic and having receptors for IgG and C3. In addition, the U937 cells have been reported to respond to lymphokines by inhibiting the growth of Toxoplasma gondii, ${ }^{20}$ and by increased levels of antibody dependent cellular cytotoxicity. ${ }^{30}$

Since we have shown that $L$. pneumophila replicates in U937 cell culture and that the increase in bacterial number over time is due to intracellular growth of the organism, the $U 937$ cell model fulfills the most basic requirements as a model for the study of $L$. pneumophila/macrophage interaction. The model also shows that growth is coincident with a loss of viability of the host cells. Moreover, we found that a derivative of strain $130 \mathrm{~b}$ that had been passaged on BCYE plates more than 200 times not only lost its ability to infect guinea-pigs, but also became unable to grow in U937 cells and cause the associated CPE. By these criteria, the U937 cells are a suitable model for the study of $L$. pneumophila/macrophage interaction.

\section{Materials and methods}

Bacteria. Maintenance of $L$. pneumophila serogroup 1, strain $130 \mathrm{~b}$ has been described. ${ }^{29}$ The bacteria $\left(10^{7}\right)$ were inoculated into the peritoneal cavities of guinea-pigs and recovered from the spleen three days later. The organisms were stored in $0.01 \mathrm{M}$ phosphate buffered saline, $\mathrm{pH}$ 7.4 (PBS) at $-70^{\circ} \mathrm{C}$ and passaged minimally on buffered charcoal yeast extract agar (BCYE). The strain was again passaged through guinea-pigs immediately prior to this study.

For each experiment several colonies were scraped off the surface of the agar and inoculated into filtered yeast extract broth as described. ${ }^{29}$ After $24 \mathrm{~h}$ on a shaking incubator at $37^{\circ} \mathrm{C}$, the culture had reached a cell density of $1-2 \times 10^{10} \mathrm{CFU} / \mathrm{ml}$. The bacteria were then harvested, washed in Dulbecco's Modified Eagle Medium (DMEM, Gibco, Grand Island, NY, U.S.A.), and used to inoculate the U937 cells.

U937 cells. U937 cells were kindly provided by Dr J. J. Marr (University of Colorado, Denver, CO, U.S.A.) and maintained as the replicative non-adherent monocyte-like cells in DMEM supplemented with $10 \%$ fetal calf serum (FCS) and $50 \mu \mathrm{g} / \mathrm{ml}$ gentamicin sulfate. Under these conditions, the doubling time of the U937 cells was typically $12 \mathrm{~h}$ (data not shown).

When the culture was at late logarithmic phase of growth (around $10^{6} \mathrm{cell} / \mathrm{s} / \mathrm{ml}$ ), the cells were diluted to $2-3 \times 10^{6} \mathrm{cells} / \mathrm{ml}$ and $12-0$-tetra decanoylphorbol-13-acetate (TPA, Sigma, St Louis, MO, U.S.A.) was added at a final concentration of $10^{-8} \mathrm{M}$ for $48-72 \mathrm{~h}$. The cells were removed from the flask with $0.2 \%$ EDTA, washed with DMEM and distributed into wells of either a 24-well or a 96-well plate (Costar, Cambridge, MA, U.S.A.) at cell densities of $5 \times 10^{5} /$ well and $2 \times 10^{5} /$ well, respectively. By examining the plates with an inverted microscope, we estimated that these densities resulted in a $50-75 \%$ covering of the surface of the plate.

Coincubation of L. pneumophila with $U 937$ cells. After the macrophages had settled onto the plates $(1-2 \mathrm{~h})$, the bacteria were added in DMEM at a ratio of $1: 1$ (bacteria:cells) and incubated for $2 \mathrm{~h}$. After three washes with $1 \mathrm{ml}$ DMEM, the cultures were incubated in DMEM/FCS. At each time point, the cells were lysed by the addition of $50 \mu \mathrm{l}$ of $2 \%$ Triton X- 
100 (final concentration of $0.1 \%$, which did not affect the viability of the bacteria). The bacterial count was determined by diluting the lysate in saline and spotting aliquots $(10 \mu \mathrm{l})$ onto BCYE plates.

An equivalent number of macrophages $\left(5 \times 10^{5} / \mathrm{ml}\right)$ were disrupted by three cycles of freezing and thawing. When stained with the vital stain Trypan blue we found that all the cells were dead. The disrupted macrophages were then incubated with $10^{3}$ organisms (without washing or incubating with antibiotics), and the number of viable bacteria was determined every $24 \mathrm{~h}$.

Cytopathic effect. The cytopathic effect (CPE) of the organisms was determined by assaying the number of viable U937 cells remaining in the wall after infection with L. pneumophila. To distinguish between living and dead 4937 cells, we used a vital stain, the tetrazolium salt 3 (4,5-dimethyl thiazol-2-yl)-2,5,-diphenyl tetrazolium bromide (MTT, Sigma, St. Louis, MO, U.S.A.), as previously described. ${ }^{22}$

To standardize the relationship between color reaction and the number of remaining viable cells, we plated serial dilutions of transformed U937 cells into 24 -well plates. We added $50 \mu \mathrm{l}$ of a stock solution of MTT $(5 \mathrm{mg} / \mathrm{ml}$ dissolved in PBS and filter sterilized) to the wells of the assay and incubated for $4 \mathrm{~h}$ at $37^{\circ} \mathrm{C}$. The supernatants were expelled and $1 \mathrm{ml}$ acid-isopropanol $(0.04 \mathrm{~N} \mathrm{HCl}$ in isopropanol) was added to each well. After thorough mixing, the optical density of each well was read at several wavelengths using acid-isopropanol as a blank. The correlation between the optical density and number of viable cells was determined by staining various numbers of live cells. A regression analysis using the method of least squares showed a high degree of correlation between staining and cell number $(r>0.99)$.

As living aerobic bacteria would also be expected to incorporate the dye, we determined the minimum concentration of $L$. pneumophila required to give absorbance under these conditions. This concentration $\left(10^{8} / \mathrm{ml}\right)$ was never attained during the growth of these organisms in the U937 cells (data not shown).

To demonstrate a cytopathic effect on the cells, we plated U937 cells onto a 96 -well plate and infected them with $L$. pneumophila at various bacteria: cell ratios. We then incubated the cultures with gentamicin sulfate $(50 \mu \mathrm{g} / \mathrm{ml})$ to kill the extracellular organisms. (By performing bacterial assays with different concentrations of gentamicin, we found that $>99 \%$ of the inoculum had been killed after $2 \mathrm{~h}$ with a concentration of $50 \mu \mathrm{g} / \mathrm{ml}$.) We then washed the cultures, incubated them in $0.1 \mathrm{ml} \mathrm{DMEM} / \mathrm{FCS}$ and at each time point we added $5 \mu \mathrm{l}$ of the stock solution of MTT as described above, reading the plates on an EAR 400 AT automatic ELISA reader (SLT Labinstruments USA, Ronkonkoma, NY) at $O D_{550}$. In some experiments the organisms were killed with gentamicin prior to adding the bacteria to the monolayers.

Production and characterization of a serogroup 1 specific monoclonal antibody. L. pneumophila were harvested from colonies grown on BCYE agar, washed in PBS, and adjusted to a concentration of $10^{10}$ bacteria $/ \mathrm{ml}$. The bacteria were then either boiled for $30 \mathrm{~min}$ or incubated for $12 \mathrm{~h}$ in $2 \%$ formaldehyde at $4^{\circ} \mathrm{C}$. The bacteria were then stored at $-70^{\circ} \mathrm{C}$. For each inoculum, the bacteria were pooled and inoculated intraperitoneally into two Balb/c mice (Jackson Laboratories, Bar Harbor, ME, U.S.A.). The first inoculation contained a total of $1 \times 10^{9}$ organisms and the subsequent inoculations, given 2 and 4 weeks after the original, contained $5 \times 10^{8}$ organisms. Four days after the last injection, the mice were sacrificed and splenic cells were fused with SP2 cells as described by Oi et al..$^{31}$ Monoclonal antibody (mAb) $1 \mathrm{C5}$ (lgG3) was found to be specific for $L$. pneumophila serogroup 1 and was purified on a Protein $A$ sepharose column (Biorad, Richmond, CA, U.S.A.) according to the manufacturer's directions.

Direct fluorescence assay of intracellular bacteria. Fluorescent labeling was performed by previously described methods. ${ }^{32}$ Briefly, $1 \mathrm{mg}$ of mAb $1 \mathrm{C} 5$ was dialysed against a $0.1 \mathrm{M}$

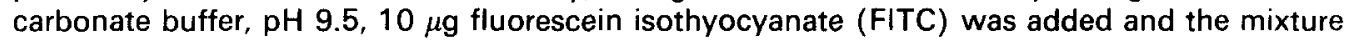
was stirred in the dark for $2 \mathrm{~h}$. The unbound FITC was removed by filter dialysis on a miniAmicon system (Danvers, MA, U.S.A.). The conjugated $1 \mathrm{C5}$ (FITC-1C5) was stored at $-70^{\circ} \mathrm{C}$ and diluted 1:100 in PBS for routine use.

TPA-treated U937 cells were plated onto $12 \mathrm{~mm}$ diameter glass coverslips, and inoculated as described in the kinetics studies. All subsequent stops were performed at room temperature. The cultures were fixed in methanol for at least $5 \mathrm{~min}$ to allow the mAb to penetrate the macrophages. The samples were then washed in PBS, and $50 \mu \mathrm{l}$ of FITC-1C5 was added for $30 \mathrm{~min}$. The samples were then washed in distilled water, air dried, and mounted onto a slide with Bacto FA mounting fluid (Difco, Detroit, MI, U.S.A.). The slides were examined with a Zeiss fluorescent microscope (Carl Zeiss Inc., New York, NY, U.S.A.) using vertical illumination, 
and photographs were taken with a Zeiss Ikon camera using T-MAX 100 film (Eastman Kodak Co, Rochester, NY, U.S.A.).

Living cultures were stained by inverting the coverslip on a drop of FITC-1 C5 and incubating $15 \mathrm{~min}$ at $37^{\circ} \mathrm{C}$ in the presence of $7 \% \mathrm{CO}_{2}$. The cultures were examined as before and photographs were taken within $15 \mathrm{~min}$.

We thank Dr J. J. Marr of the University of Colorado for providing the U937 cells, Dr Lathe Claflin of this department for the use of the ELISA reader, and Drs Garth Jones and Rolf Freter, also of this department, for the use of the fluorescent microscope. This work was supported by Public Health Service grant Al24731 and by the Veterans Administration. N.C.E. is supported by a Veterans Administration Career Development Award.

\section{References}

1. Blackmon JA, Hicklin MD, Chandler FW, et al. Legionnaires' disease: pathological and historical aspects of a 'new' disease. Arch Path Lab Med 1978; 102: 337-43.

2. Davis GD, Winn WC, Jr, Gump DW, Beaty HN. The kinetics of early inflammatory events during experimental pneumonia due to Legionella pneumophila in guinea pigs. J Infect Dis 1983; 148: 82335.

3. Horwitz MA, Silverstein SC. Legionnaires' disease bacterium (Legionella pneumophila) multiplies intracellularly in human monocytes. J Clin Invest 1980; 66: 441-50.

4. Holden EP, Winkler HH, Wood DO, Leinbach ED. Intracellular growth of Legionella pneumophila within Acanthamoeba caste/lanii neff. Infect Immun 1984; 45: 18-22.

5. Newsome AL, Baker RL, Miller RD, Arnold RR. Interactions between Naegleria fowleri and Legionella pneumophila. Infect Immun 1985; 50: 449-52.

6. Fields BS, Barbaree EB, Shotts EB Jr, et al. Comparison of guinea pig and protozoan models for determining virulence of Legionella species. Infect Immun 1986; 53: 553-9.

7. Daisy JA, Benson CE, McKitrick J, Friedman HM. Intracellular replication of Legionella pneumophila. J Infect Dis 1981; 143: 460-4.

8. Oldham LJ, Rodgers FG. Adhesion, penetration and intracellular replication of Legionella pneumophila: an in vitro model of pathogenesis. J Gen Microbiol 1985; 131: 697-706.

9. Jacobs RF, Locksley RM, Wilson CB, Haas JE, Klebanoff SJ. Interaction of primate alveolar macrophages and Legionella pneumophila. J Clin Invest 1984; 73: 1515-23.

10. Kishimoto RA, Kastello MD, White JD, et al. In vitro interaction between normal cynolmolgus monkey alveolar macrophages and Legionnaires' disease bacteria. Infect Immun 1979; 25: 761-3.

11. Kishimoto RA, White JD, Shirey FG, et al. In vitro response of guinea pig peritoneal macrophages to Legionella pneumophila. Infect Immun 1981; 31: 1209-13.

12. Nash TW, Libby DM, Horwitz MA. Interaction between the Legionnaires' disease bacterium (Legione/la pneumophila) and human alveolar macrophages. J Clin Invest 1984; 74: 771-82.

13. Yamomoto Y, Klein TW, Newton CA, Widen R, Friedman H. Growth of Legionella pneumophila in thioglycolate-elicited peritoneal macrophages from A/J mice. Infect Immun 1988; 56: 370-5.

14. Yoshida S, Mizuguchi Y. Multiplication of Legionella pneumophila Philadelphia-1 in cultured peritoneal macrophages and its correlation to susceptibility of animals. Can J Microbiol 1986; 32: 438-42.

15. Sundstrom C, Nilsson K. Establishment and characterization of a human histiocytic lymphoma cell line (U937). Int J Cancer 1976; 17: 565-77.

16. Harris P, Ralph P. Human leukemic models of myelomonocytic development: a review of the HL-60 and U937 cell lines. J. Leukocyte Biol 1985; 37: 407-22.

17. Radzun HJ, Parwaresch MR, Sundstrom C, Nilsson K, Eissner M. Monocitic origin of the human hematopoietic cell line U937 and its convertibility to macrophages evidenced by isoenzyme mapping. Int J Cancer 1983; 31: 181-6.

18. Gilbert D, Peulve P, Daveau M, Ripoche J, Fontaine M. Modulation of complement receptors of a human monocyte cell line, U937, during incubation with phorbol myristate acetate: expression of an iC3bspecific receptor (CR\#). Eur J Immunol 1985; 15: 986-91.

19. Looker DL, Martinez S, Horton JM, Marr JJ. Growth of Leishmania donovani amastigotes in the continuous human macrophage cell line U937: studies of drug efficacy and metabolism. $J$ Infect Dis 1986; 154: 323-7.

20. Wing EJ, Koren HS, Fisher DG, Kelley V. Stimulation of a human macrophage-like cell line (U937) to inhibit replication of an intracellular pathogen. J Reticuloendoth Soc 1981; 29: 321-9.

21. Folks TM, Justement J, Kinter A, Dinarello CA, Fauci AS. Cytokine-induced expression of HIV-1 in a chronically infected promonocyte line. Science 1987; 238: 800-2.

22. Green LM, Reade JL, Ware CF. Rapid colorimetric assay for cell viability: application to the quantitation of cytotoxic and growth inhibitory lymphokines. J Immunol Met 1984; 70: 257-68.

23. Mandell GL. Interaction of intraleucocytic bacteria and antibiotics. J Clin Invest 1972; 52: 1673-9. 
24. Elliott JA, Johnson W. Virulence conversion of Legionella pneumophila serogroup 1 by passage in guinea pigs and embryonated eggs. Infect Immun 1982; 35: 943-6.

25. McDade JE, Shepard CC. Virulent to avirulent conversion of Legionnaires' disease bacterium (Legione/la pneumophila) - its effect on isolation techniques. J Infect Dis 1979; 139: 707-11.

26. Horwitz MA. Characterization of avirulent mutant Legionel/a pneumophila that survive but do not multiply within human monocytes. J Exp Med 1987; 166: 1310-28.

27. Engleberg, NC, Pearlman E and Eisenstein BI. Legionel/a pneumophila surface antigens cloned and expressed in Escherichia coli are translocated to the host cell surface and interact with specific antiLegionel/a antibodies. J Bacteriol 1984; 160: 199-203.

28. Engleberg NC, Pearlman E, Dixon D and Eisenstein BI. Antibodies isolated using cloned surface antigens recognize antigenically related components of Legionella pneumophila and other Legionella species. J immunol 1986; 136: 1415-17.

29. Pearlman E, Engleberg NC, Eisenstein BI. Identification of protein antigens of Legionel/a pneumophila serogroup 1. Infect Immun 1985, 47: 74-9.

30. Larrick JW, Fischer DG, Anderson SJ, Koren H. Characterization of a human macrophagelike celf line stimulated in vitro: a model of macrophage functions. J Immunol 1980; 125: 6-12.

31. Oi VT, Herzenberg LA. Immunoglobulin-producing hybrid cell lines. In: BB Mishell and SM Shiggi, eds. Selected methods in cellular immunology. San Francisco. WH Freeman and company, 1982; 351-72.

32. Hurn BAL, Chantler SM. Production of reagent antibodies. In: Van Vunakis H, Langone JJ, eds. Methods in enzymology vol. 30: Immunochemical techniques. Academic Press Ltd, 1980. 105-6. 